\title{
A Proposal for Financially Sustainable Population Health Organizations
}

\author{
William A. Yasnoff, MD, PhD, Edward H. Shortliffe, MD, PhD, \\ and Stephen M. Shortell PhD, MPH, MBA ${ }^{3}$
}

$\mathbf{R}$ ECONFIGURING OUR HEALTH delivery sector to provide safe and effective care, improve health, and simultaneously reduce costs requires both efficient, high-quality medical services and community-wide population health and prevention activities, supported by the immediate availability of comprehensive electronic patient information. ${ }^{1}$ Given the various health care stakeholders in our communities, who often have divergent interests and incentives, how can we establish and sustain organizations that can simultaneously achieve all of these goals?

Shortell $^{2,3}$ has proposed entities that might be called population health organizations (PHOs) and that would focus on addressing all of the determinants of health in a community, integrating the medical, public health, and community/social service sectors. These coordinating organizations would assume responsibility and accountability for a defined community-wide geographic population that would extend beyond any individual Accountable Care Organization's (ACO's) or integrated delivery system's own group of patients. They would engage leaders in health care delivery, public health, education, housing, labor, and related sectors to develop effective cross-cutting services to meet the health needs of the entire community. Targeting the entire population would ensure that long-term prevention projects are included because all stakeholders would benefit, regardless of ongoing and inevitable shifts in the organizational affiliations of individuals in the community. Difficult, multifaceted problems with broad impact can be addressed, such as obesity, diabetes, and coronary artery disease. Risk-adjusted global or capitated payments from Medicare, Medicaid, and commercial carriers would create budgets tied to achieving predefined quality and costperformance metrics.

Two key prerequisites for the success of PHOs are information systems that can provide comprehensive electronic medical information for every individual (to support targeting of interventions) and ongoing funding sufficient to maintain and enhance both the information infrastructure and prevention activities over time. Yasnoff et $\mathrm{al}^{4}$ have proposed health record banks (HRBs) as an effective mechanism for assuring the availability of comprehensive electronic patient information in communities. HRBs are trusted community organizations that serve as custodians of a comprehensive integrated copy of each member's personal, private health information, including both medical records and personal data that the patient may opt to add. They also could include community-wide environmental health, housing, labor, and related human services data. The patient explicitly controls who may access which parts of the information in his or her individual account. ${ }^{5,6}$ When patients seek care, they give permission to their health care providers to access some or all of their up-to-date health records available through the HRB. At the patient's request, when care is complete, the new records from that visit or hospitalization are securely deposited into the HRB and made available for future use. This approach solves the problems of privacy (through patient control), stakeholder cooperation (because the patients request their own records, the Health Insurance Portability and Accountability Act [HIPAA] regulations require every stakeholder to provide them electronically if they are available in that form), and financial sustainability (with revenue from optional applications, or "apps," for use by patients as well as use of their data for research purposes, but only with their permission), and it coexists with providers keeping their own local copies of records.

HRBs can promote greater availability of electronic information by subsidizing electronic health records (EHRs) for outpatient physicians, thereby assuring much higher levels of adoption resulting in more complete information for each patient. They also can generate substantial net revenue from apps, advertising, and fees for data access for research or other purposes (with patient permission) above and beyond their own costs of operation. For example, a "peace of mind" app that automatically notifies loved ones when an emergency physician accesses HRB records (meaning that the person is undergoing emergency treatment) is a compelling value to consumers and their families for which they likely would be willing to pay a modest fee. With consumer permission, HRBs could readily identify eligible patients for clinical trials as well as provide data for use by researchers and policy makers. By sharing part of the revenue generated from these research applications on a pro rata basis with account holders, consumers also would have an incentive to allow their data to be used for these purposes. The HRB

\footnotetext{
${ }^{1}$ NHII Advisors, Arlington, VA, and Division of Health Sciences Informatics, Johns Hopkins University, Baltimore, MD.

${ }^{2}$ College of Health Solutions, Arizona State University, Phoenix, AZ.

${ }^{3}$ Division of Health Policy and Management, School of Public Health, University of California, Berkeley.
} 
revenue could be used not only for information system upgrades but also to support and expand community-wide prevention and population health activities.

Although the overall financial viability of the business model for HRBs has yet to be demonstrated, each individual component is derived from available evidence. ${ }^{7}$ The willingness of physicians to accept free or subsidized EHRs in exchange for depositing information in an HRB (required by HIPAA upon patient request), and establishing an office policy that all patients must have an HRB account, was confirmed by a letter of intent to participate from a $1000+$ physician group. Another $500+$ physician group also indicated its readiness. ${ }^{7}$ Because Halamka has previously reported 93\% patient acceptance of free online access to a single institution's EHR when recommended by physicians, ${ }^{8}$ a similar rate of acceptance is anticipated for HRB enrollment. Finally, in a market research survey of a stratified random sample of over 1000 online users, $20 \%$ of respondents would purchase unspecified reminder services, with $17 \%$ of overall respondents willing to spend more than $\$ 50 /$ year. $^{7}$

Combining a PHO and HRB into what we call a Health and Prevention Promotion Initiative (HAPPI) results in a financially self-sustaining entity that can deliver population health and prevention services as well as comprehensive electronic patient records for the community. The PHO organizes and executes prevention and population health activities, while the integrated HRB provides the necessary patient record information. The revenue generated can be an added source of support for the PHO, along with initial contributions by stakeholders including community and provider organizations. Once started, a HAPPI can be internally self-sufficient without the need for additional reimbursement or outside funding.

The HAPPI also helps to align the incentives for all relevant stakeholders. Health plans, hospitals, and ACOs get the benefits of ongoing population health and prevention efforts in the community, support for care coordination, and the efficiencies in care that result from the availability of comprehensive electronic records for each patient. Physicians benefit from the availability of permanent, ongoing EHR subsidies and more complete patient information from the HRB, which will be particularly helpful in managing patients with several complex chronic illnesses that involve multiple providers and settings of care. Comprehensive patient records also provide the basis for timely clinical decision support, and the availability of pooled data across large numbers of patients enables a learning health system. ${ }^{9}$ Patients benefit from lower costs and better care, and also have better opportunities for engagement through the availability of their own comprehensive health records, with privacy protected through their own control of access by others. Payers benefit through lower costs, better care, and the availability of timely data for payment and policy development. The research community benefits from the greater availability of detailed electronic patient information, accessible with patient consent.

\section{Conclusion}

Financially self-sufficient HRBs provide the information infrastructure needed for PHOs to succeed. Together they help promote both improved care and sustainable prevention, while providing new and compelling value to patients, providers, payers, researchers, and policy makers. The time is ripe for communities that have PHO potential to implement the HRB concept simultaneously to complement and support their PHO efforts.

\section{Author Disclosure Statement}

Drs. Yasnoff, Shortliffe, and Shortell declared no conflicts of interest with respect to the research, authorship, and/ or publication of this article. The authors received no financial support for this article.

\section{References}

1. Huang X, Rosenthal MB. Transforming specialty practicethe patient-centered medical neighborhood. N Engl J Med. 2014;370:1376-1379.

2. Shortell SM. Bridging the divide between health and health care. JAMA. 2013;309:1121-1122.

3. Shortell SM. A bold proposal for advancing population health. http://www.iom.edu/Global/Perspectives/2013/Bold Proposal.aspx. Accessed May 5, 2014.

4. Yasnoff WA, Sweeney L, Shortliffe EH. Putting health IT on the path to success. JAMA. 2013;309:989-990.

5. Health Record Banking Alliance. HIE Architecture white paper. http://www.healthbanking.org/docs/HRBA\%20Arch itecture \%20White\%20Paper\%20Jan\%202013.pdf. Accessed May 5, 2014.

6. Health Record Banking Alliance. Business Model white paper. http://www.healthbanking.org/docs/HRBA\%20Business\% 20Model\%20White\%20Paper\%20Dec\%202012.pdf. Accessed May 5, 2014.

7. Yasnoff WA, Shortliffe EH. Lessons learned from a health record banking start-up. Meth Inf Med. 2014;53:66-72.

8. Halamka J. Standards Town Hall: Using Harmonized Standards in the Era of Meaningful Use. http://www.prolibraries $. \mathrm{com} / \mathrm{himss} /$ ? select $=$ session\&sessionID $=686$. Accessed May 21, 2014.

9. Smith M, Saunders R, Stuckhardt L, McGinnis JM, eds; Committee on the Learning Health Care System in America; Institute of Medicine. Best Care at Lower Cost: The Path to Continuously Learning Health Care in America. Washington, DC: National Academies Press; 2013.

Address correspondence to: William A. Yasnoff, MD, PhD NHII Advisors 1854 Clarendon Boulevard Arlington, VA 22201

E-mail: william.yasnoff@nhiiadvisors.com 